\title{
Relationship between maternal pathology and infant social withdrawal: Analysis of a 268-outpatient population
}

\author{
Maria Lucia Maulucci ${ }^{1}$, Vincenzo Currò ${ }^{1}$, Silvia Maulucci ${ }^{1},{\text { Emilia de } \text { Rosa }^{1} \text {, Livia De Giovanni }}^{2}$ \\ ${ }^{1}$ Department of Neuroscience, Catholic University Hospital, Rome, Italy \\ ${ }^{2}$ Statistic Department, Lumsa University, Rome, Italy \\ Email: lucia.maulucci@libero.it
}

Received 2 May 2013; revised 3 June 2013; accepted 12 June 2013

Copyright (C) 2013 Maria Lucia Maulucci et al. This is an open access article distributed under the Creative Commons Attribution License, which permits unrestricted use, distribution, and reproduction in any medium, provided the original work is properly cited.

\begin{abstract}
Objective: Sustained withdrawal is a sign of infant distress and may be determined by extreme conditions like organic pathology or relationship problems. The Alarm Distress Baby Scale is the best instrument to identify withdrawal in infants between 2 and 24 months of age. The aim of this study was to assess the association between infant social withdrawal behavior and maternal pathology in the perinatal period. Method: The target sample of the study was 134 mother-child couples examined at the Policlinico Agostino Gemelli General Pediatric Outpatient Clinic and Psychiatric Outpatient Clinic, in Rome. We analyzed the relationship between withdrawal behavior and psychological and organic prenatal/postnatal pathology, using the Chi Square exact test. Results: When the mother suffers from both organic and psychological pathologies in the perinatal period, the risk of withdrawal is increased: the probability of infant withdrawal behavior is increased by maternal organic postnatal pathology by 14 times, by maternal psychological postnatal pathology by 9 times, and by maternal psychological postnatal depression, measured by Edinburgh Postnatal Depression Scale, by almost 4 times. Conclusion: Increased or sustained withdrawal reactions can be observed in unsatisfactory mother-child interaction. Maternal risk factors should be strictly considered in the pediatric clinical evaluation.
\end{abstract}

Keywords: Maternal Pathology; Infant Social Withdrawal Early Prevention; Alarm Distress Baby Scale

\section{INTRODUCTION}

Infants are born with an ability to be aware of the world around them as they show immediate connection to human beings soon after birth. The interaction skills include the ability to initiate and maintain eye contact, to vocalize and to use facial expression and body movements to engage others' interaction. In newborn clinical evaluation, pediatricians have always emphasized the newborn's unique behavioral capacities, Brazelton [1], and the "vital tone" as defined by Pierre Marty [2]. This is a clear sign of good health and part of pre-verbal social communication. The infant's ability to interact with the others increases enormously within the first two years of life and is reinforced by lallation: the latter develops from the second month of life and makes possible the development of thought and symbolic verbal communication.

In a situation of danger, as the child feels powerless, he implements automatic defensive reactions that are common to primate and consist of mobilizing genetically preprogrammed "attack-escape" and "conservation-withdrawal" responses [3]. Engel describes infant withdrawal as a conservation system and a "biological threshold mechanism, where organism survival is supported by the process of disengagement and inactivity, vis-a-vis the external environment" [4].

Transient social withdrawal is a normal aspect of infant behavior. However, if it becomes persistent in the form of diminished eye contact, facial expression, smiling, cooing, body movements, withdrawal has not to be underestimated, but considered as a very important alarm signal of developmental disorders and others psychological pathologies [5].

Sustained infant withdrawal is observed in several clinical situations such as fever, dehydration, post-seizure critical state, intoxication, central nervous system diseases, visual and auditory sensory impairment [6]; in severe forms of protein deficiency malnutrition, such as Kwashiorkor, withdrawal is intense and prolonged, and its abatement is a reliable sign of recovery [7-9]; with- 
drawal is also a feature of nonorganic failure to thrive [10-12].

Furthermore, systems of biological response to danger are regarded as the precursors of anxiety and depression. In a study on children separated from their mothers, Spitz et al. introduced the term "withdrawal" as a key feature of anaclitic depression [13]. Withdrawal is a key symptom of autism, pervasive developmental disorders, anxiety, infantile depression and is an element in the clinical picture of attachment disorganization [14] and post-traumatic stress disorder [15]. Withdrawal behavior is a part of the symptomatology of several DC 0 - 3 R diagnoses [16], as Post-Traumatic Stress Disorder (100), Deprivation/Maltreatment Disorder (250), Disorders of Affect (200), Prolonged Bereavement/Grief Reaction (210), Anxiety, Disorders of Infancy and Early Childhood (220), Depression of Infancy and Early Childhood (230), Regulation Disorders of Sensory Processing (400) and Multisystem Developmental Disorder (700).

Moreover, infant withdrawal can be a consequence of maternal depression [17]. Robertson and Bowlby [18] in their observations on infants suffering from maternal deprivation described three different stages in the infant reaction: Protest, Despair and Withdrawal. Fraiberg [19] described the Freezing behavior as an early defense mechanism arising from the age of four months, when the child is faced with a strong experience of helplessness. This was then described by Beebe [20] as the precursor of attachment disorganization.

The Alarm Distress Baby Scale, ADBB, is a practical and accurate tool to identify sustained withdrawal behavior in children between 2 to 24 months of age [21] in different settings and different cultures. This scale could be used by nurses, psychologists and physicians after a short period of training; its administration requires 10 15 minutes.

ADBB evaluates social behaviors organized into eight items: 1) Facial Expression; 2) Eye Contact; 3) General Level of Activity; 4) Self-Stimulating Gestures; 5) Vocalizations; 6) Response to Stimulation; 7) Relationship; 8) Attraction. Each item is rated from 0 to 4 (0: no unusual behavior; 4: severe unusual behavior); the total score could range from 0 (normal) to 32 (severe withdrawal). An important point about trans-cultural validity is that five studies found the same cut-off score of 5. The scale has been shown to have good reliability and validity $[3,22]$.

Our research team conducted the Italian validation, which showed a cut-off score of 5 and the best sensitivity/specificity ratio of 0.82 versus 0.95 [23]. We noticed an interesting correlation between maternal postnatal depression and positive ADBB, but no correlation in mothers who suffered from serious organic pathologies arisen before pregnancy. We wondered if suffering from either organic or psychological pathologies in the perinatal period could be significantly correlated with infant withdrawal. In psychology, the perinatal period goes from conception to the second year of age [24].

Our hypothesis is that an infant withdraws only if the maternal pathology involves the parenthood process, which is the mother-infant relationship.

We had to elaborate on this aspect since there are no studies in the literature that examine this issue. This study could bring a remarkable contribution in the prevention, diagnosis and treatment of infant withdrawal.

\section{METHOD}

The target sample of the study was 134 mother-child couples examined at the Policlinico Agostino Gemelli General Pediatric Outpatient Clinic and Psychiatric Outpatient clinic, Rome.

Mothers and children involved in the study included those who arrived for first routine check-up in the wellbaby clinic, as well as mothers asking for psychiatric consultation for a specific psychological problem.

We included all infants between two and twenty-four months of age, both male and female, born either from a single or a twin birth, conceived either naturally or by artificial insemination, adopted, belonging to any race or nationality.

We excluded infants suffering from acute pathologies, from neurological problems, and premature children.

We didn't apply any exclusion criteria for mothers.

\subsection{Instruments}

Demographics of mothers: using a perinatal biographical questionnaire, we obtained information about age, education, marital status, with emphasis on the presence of maternal pathology or stressful life events during the perinatal period.

Edinburgh Postnatal Depression Scale (EPDS): a paper-pencil self-reporting questionnaire; a score $\geq 12$ indicates maternal postnatal depression risk.

Alarm Distress Baby Scale (ADBB): a specific instrument to assess sustained withdrawal behavior in infants between two and twenty four months of age. A score $\geq 5$ indicates infant withdrawal risk.

Perinatal consultation: a psychological evaluation focused on the maternal difficulties and suffering.

We analyzed the relationship between withdrawal behavior and psychological and organic prenatal/postnatal pathology. The variables considered for the analysis were: 1) for the mothers, the presence/absence (codified with $1 / 0$ ) of psychological prenatal/postnatal pathology, the presence/absence (codified with 1/0) of organic prenatal/ postnatal pathology, the presence/absence (codified with $1 / 0$ ) of psychological postnatal depression risk measured 
by EPDS (presence $=$ EPDS score $\geq 12$ ); 2 ) for the infant, the presence/absence (codified with $1 / 0$ ) of withdrawal behavior ( presence $=$ ADBB score $\geq 5$ ).

\subsection{Statistics}

We used Chi Square exact test of dependence between withdrawal behavior, psychological/organic prenatal/ postnatal pathology and psychological postnatal depression risk measured by EPDS; logistic regression analysis of withdrawal behavior as a dependent variable and psychological and organic prenatal/postnatal pathology and psychological postnatal depression risk measured by EPDS as independent variables.

\section{RESULTS}

Mother, father and infant demographics are reported in Tables 1-3, respectively. Mother clinical characteristics are reported in Table 4. The results of tests of dependence for ADBB are reported in Table 6.

Mother postnatal pathologies, either organic or psychological, have a significant association with infant withdrawal behavior $(p<0.05)$. In particular, the relative frequency of infants with withdrawal behavior was significantly higher when the mother suffered from either organic or psychological postnatal pathologies.

A binary logistic regression analysis was applied to investigate how infant withdrawal behavior could be predicted by the presence of one or more pathologies of the mothers. The model identified three variables over the five for the prediction of withdrawal behavior. The variables are postnatal pathologies, either organic or psychological and psychological postnatal depression risk measured by EPDS.

The maximum likelihood estimates of the parameters of the logit model are presented in Table 5. The likelihood ratio test confirms the validity of the model.

The analysis of the odds ratio shows that the probability of infant withdrawal behavior is increased by maternal organic postnatal pathology by 14 times, by maternal psychological postnatal pathology by 9 times, and by maternal psychological postnatal depression risk measured by EPDS by nearly 4 times.

\section{DISCUSSION}

Withdrawal behavior as a defense mechanism to safeguard survival is implemented as a signal of distress behavior by human infant as well as other young primates.

The relationship between maternal depression and anxiety during pregnancy has a negative effect on infant behavior and development. Infants of mothers who report to be more depressed or anxious since childbirth have significantly higher ADBB scores [22,23]. These studies confirmed the correlation between maternal psychologi-
Table 1. Mothers' demographic characteristics.

\begin{tabular}{ccc}
\hline Total number & & 134 \\
\hline Nationality (Non Italian/Italian) & & $35 / 99$ \\
Mean Age (133 subjects) & & 34,89 \\
& Primary & 2 \\
Educational Level (133 subjects) & Middle & 17 \\
& High & 64 \\
& Degree & 46 \\
Marital Status & Single & 3 \\
& Married & 102 \\
Birth order & Non-married & 26 \\
& First born & 77 \\
Mourning (61 subjects) & Second born & 49 \\
& Third born and over & 19 \\
Abortions (115 subjects) & & 12 \\
Medications (83 subjects) & Voluntary & 6 \\
& & 22 \\
\hline
\end{tabular}

Table 2. Parents' characteristics.

\begin{tabular}{ccc}
\hline Mean age (131 subjects) & & 37,87 \\
& Primary & 1 \\
Educational level (123 subjects) & Middle & 28 \\
& High & 60 \\
& Degree & 34 \\
\hline
\end{tabular}

Table 3. Parents' characteristics.

\begin{tabular}{ccc}
\hline Mean birth weight (130 subjects) & & 3279 gr. \\
Mean gestational age (109 subjects) & & 38,85 weeks \\
& Cesarean & 46 \\
Delivery (130 subjects) & Spontaneous & 75 \\
& Breastfeeding & 84 \\
Nursing & Bottle feeding & 16 \\
& Mixed & 41 \\
Twin birth & Heterozygotic & 2 \\
& Monozygotic & 0 \\
\hline
\end{tabular}

Table 4. Mothers' clinical characteristics.

\begin{tabular}{cc}
\hline Control group & 49 subjects \\
Psychological pathology in the past & 19 subjects \\
$\begin{array}{c}\text { Postnatal depression/perinatal } \\
\text { psychological pathologies } \\
\text { High EPDS score }\end{array}$ & 11 subjects \\
Organic pathology in the past & 22 subjects \\
$\begin{array}{c}\text { Organic pathology arisen } \\
\text { in the perinatal period } \\
\text { Stressful life events }\end{array}$ & 17 subjects \\
\hline
\end{tabular}

cal pathology and infant withdrawal behavior. However also mothers suffering from an organic disease triggered social withdrawal in their infants, if the organic or psychological pathology occurred during the perinatal period. 
Table 5. Estimates of parameters of the logit model and related $p$-values.

\begin{tabular}{ccccc}
\hline & Beta coefficient & $\begin{array}{c}\text { Standard } \\
\text { error }\end{array}$ & $p$-value & $\exp$ (beta) \\
\hline $\begin{array}{c}\text { Organic postnatal } \\
\text { pathology }\end{array}$ & 2.651 & 0.693 & 0.000 & 14.175 \\
$\begin{array}{c}\text { Pshycological } \\
\text { postnatal pathology }\end{array}$ & 2.233 & 0.710 & 0.001 & 9.327 \\
EPDS & 1.353 & 0.689 & 0.049 & 3.860 \\
Constant & -2.015 & 0.371 & 0.000 & 0.133 \\
Likelihood ratio test & & & 0.000 & \\
\hline
\end{tabular}

Table 6. Test of dependence for ADBB.

\begin{tabular}{cc}
\hline & Chi Square $p$-value \\
\hline Organic prenatal pathology/ADBB & 0.130 \\
Organic postnatal pathology/ADBB & 0.001 \\
Psychological prenatal pathology/ADBB & 0.259 \\
Psychological postnatal pathology/ADBB & 0.029 \\
EPDS/ADBB & 0.120 \\
\hline
\end{tabular}

In fact, infants of mothers that before pregnancy suffered from depression, obsessive syndrome, or personality disorders do not have higher ADBB scores.

In our study we divided our patient population into organic or psychological pathology, and, furthermore, into diseases which arose before or after the perinatal period.

The period of outbreak has proved to be a key point. Our results have shown that any type of pathology that occurred outside the perinatal period did not interfere with the mother-child interaction or with the process of parenthood construction. This was true even if the mothers had suffered from chronic diseases, including severe ones, regardless if these were organic or psychological (lymphomas, hemorrhages, mental or personality disorders).

A maternal pathology that occurs in the perinatal period (gestational diabetes, eclampsia, pulmonary failure, depression in pregnancy, postnatal depression, psychosis, etc.), can become a traumatic factor that upsets the process of filiation.

The mother attention drops and focuses on her suffering self; the affective investment is far from involving the child, who feels the affective deprivation and returns to a state of primary isolation using defensive withdrawal.

In our study, we observed that the probability of withdrawal is 14 times higher if the mother suffers from organic postnatal pathology than if the mother has no organic pathology. On the other hand, the presence of mother psychological postnatal pathology increases the probability of withdrawal behavior by a factor of about 9 . The presence for the mother of postnatal depression risk measured by EPDS increases the probability of withdrawal behavior by a factor of about 3 .
The influence of mother depression as measured by EPDS has only little influence on infant withdrawal behavior as shown by the $p$-values of the association tests and by the value and significance of the related estimated parameter of logit regression (Tables $\mathbf{4}$ and 5); the probability of withdrawal in the presence of postnatal depression risk measured by EPDS is almost the same as the probability of non withdrawal (odds $(0,0,1)=0.52$ ). This feature has already been described in $[25,26]$. EPDS is a self-report scale, hence not a trustworthy index of postnatal depression, unless it is followed by psychiatric assessment. Indeed, high scores may occur in patients reporting as excessive and exaggerated transient mood impairment.

We think there is a very high correlation between maternal depression and social withdrawal. The diagnosis is based not only on self-report assessment but also on clinical evaluation.

\section{Limitations of the Study}

This is study is supposed to be the first in his category. That is the reason why we could not compare the results with similar studies. Moreover, we have not considered the correlation between ADBB and the quality of caregiver-child relationship and relationship disorders, using tools as PIPE or PIR-GAS (the Parent-Infant Relationship Global Assessment Scale).

\section{CONCLUSION}

Parent-infant interaction can easily lose harmony, resulting in a loss of expectations from the infant who responds with defensive withdrawal. Sustained social withdrawal is an important indicator, since its presence indicates that the child has found no way out of a difficult situation. Sometimes, parents are not able to recognize or report the problems of their infants, especially if the mother suffers from organic or psychological pathology. The use of direct infant observation with ADBB increases the possibility to detect infant withdrawals. In our study, we observed a high association between maternal pathology and infant withdrawal. We should consider training pediatricians to learn the ADBB and referring the infant with sustained withdrawal behavior to child psychiatrist for clinical evaluation and treatment of mother-infant relationship.

\section{REFERENCES}

[1] Brazelton, T.B. (1973) Neonatal behavioral assessment scale. Clinics in Developmental Medicine, WM. Heinemann Medical Books, London.

[2] Marty, P. (1968) La depression essentielle. Revue Française de Psychanalyse, 33, 395-603. 
[3] Dollberg, D., Feldman, R., Keren, M. and Guedeney, A. (2006) Sustained withdrawal behavior in clinic-referred and nonreferred infants. Infant Mental Health Journal, 27, 292-309. doi:10.1002/imhj.20093

[4] Engel, G.L and Schmale, A.H. (1972) Conservation withdrawal: A primary regulatory process for organismic homeostatas. Physiology, emotion and psychosomatic illness. Excerpta Medica CIBA Foundation, Amsterdam, 57-85.

[5] Guedeney, A., Foucault, C., Bougen, E., Larroque, B. and Mentré, F. (2008) Screening for risk factors of relational withdrawal behaviour in infants aged $14-18$ months. European Psychiatry, 23, 150-155. doi:10.1016/j.eurpsy.2007.07.008

[6] Behrman, R.E., Vaughan, V. and Nelson, W. (1983) Nelson textbook of pediatrics. W.B. Saunders, Philadelphia.

[7] Guedeney, A. (1987) Les aspects psychosomatiques des malnutritions proteinocaloriques de la première enfance. Psychiatrie de l'Enfant, 1, 155-190.

[8] Guedeney, A. (1995) Kwashiorkor depression and attachment disorders. Lancet, 346, 1293. doi:10.1016/S0140-6736(95)91890-6

[9] Guedeney A. (1997) From early withdrawal reaction to infant depression. A baby alone does exist. Infant Mental Health Journal, 18, 339-349. doi:10.1002/(SICI)1097-0355(199724)18:4<339::AID-I MHJ2>3.0.CO;2-G

[10] Powell, G.F. and Low, J. (1983) Behavior in nonorganic failure to thrive. Developmental and Behavioral Pediatrics, 4, 26-33. doi:10.1097/00004703-198303000-00006

[11] Powell, G.F. and Bettes, B.A. (1992) Infantile depression, non organic failure to thrive, and DSM-III-R: A different perspective. Child Psychiatry and Human Development, 22, 185-198. doi:10.1007/BF00705891

[12] Menahem, S. (1984) Possible conservation withdrawal reaction in two infants. Developmental and Behavioral Pediatrics, 5, 361-363. doi:10.1097/00004703-198412000-00014

[13] Spitz, R.A. (1946) Anaclitic depression. Psychoanalytical Study of the Child, 2, 313-341.

[14] Solomon, J. and George, C. (1999) Attachment disorganization. Guilford, New York.

[15] Zeanah, C.H. (1993) Handbook of infant mental health. Guilford, New York.
[16] Zero To three (2005) National Center for Infant Clinical Programs. Diagnostic classification of mental health and developmental disorders of infancy and early childhood. Zero To Three Press, Washington.

[17] Murray, L. and Cooper, P.J. (1997) Postpartum depression and child development. Guilford, New York.

[18] Robertson, J. and Bowlby, J. (1952) Responses of young children to separation from their mothers. Courrier de la Centre International de l'Enfance, 2, 131-142.

[19] Fraiberg, S. (1982) Pathological defenses in infancy. The Psychoanalytical Quarterly, 4, 612-635.

[20] Beebe, B., Jaffe, J., Buck, K., Chen, H., Cohen, P., Feldstein, S. and Andrews, H. (2007) Six-week postpartum maternal depressive symptoms and 4-month mother- infant self- and interactive contingency. Infant Mental Health Journal, 29, 442-471. doi:10.1002/imhj.20191

[21] Guedeney, A. and Fermanian, J. (2001) A validity and reliability study of assessment and screening for sustained withdrawal reaction in infancy: The alarm distress baby scale. Infant Mental Health Journal, 22, 559-575. doi:10.1002/imhj.1018

[22] Matthey, S., Guedeney, A., Starakis, N. and Barnett, B. (2005) Assessing the social behaviour of infants: Use of the ADBB Scale and relationship to mother's mood. Infant Mental Health Journal, 26, 442-458.

[23] De Rosa, E., Currò, V., Wendland, J., Maulucci, S., Maulucci, M.L. and De Giovanni, L.(2010) Propriétés psichométriques de l'échelle Alarme Détresse Bébé (ADBB) appliquée à 81 enfants italiens. Devenir, 22, 209-223. doi:10.3917/dev.103.0209

[24] Missonier, S. (2003) La consultation thérapeutique périnatale. Éditions érès.

[25] Mellier, D., Rochette, J., Coulet, P., Grisi, S., Marguier L., Marandet, A. and Lefebvre, I. (2007) The ADBB scale used in preventive strategies for working in network between baby clinic and child mental health service. Devenir, 19, 81-108.

[26] Hartley, C., Pretorius, K., Mohamed, A., Laughton, B., Madhi, S., Cotton, M.F., Steyn, B. and Seedat, S. (2010) Maternal postpartum depression and infant social withdrawal among human immunodeficiency virus (HIV) positive mother-infant dyads. Psychology, Health \& Medicine, 15, 278-287. 